\title{
Contribución de la evaluación educativa para la formación democrática y transformadora de estudiantes
}

\author{
Contribution of the Educational Evaluation for the Democratic and Transformative \\ Formation of the Student
}

\author{
Contribuição da avaliação educacional para a formação democrática e \\ transformadora de estudantes
}

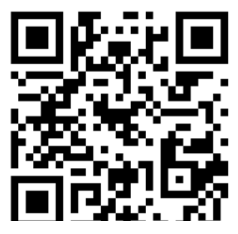

daniel.rios@usach.cl

Universidad de Santiago de Chile

Santiago, Chile

s.//orcid.org/0000-0001-6226-4499

David Herrera-Araya

Universidad de Santiago de Chile

Santiago, Chile

david.herrera@usach.cl

iD https://orcid.org/0000-0002-7631-9283

Recibido • Received • Recebido: 22 / 10 / 2019

Corregido • Revised • Revisado: 21 / 06 / 2021

Aceptado • Accepted • Aprovado: 12 / 08 / 2021

\begin{abstract}
Resumen:
Propósito. En este artículo se analiza la evaluación educativa como un campo de conocimiento teórico-práctico para la formación democrática y transformadora del estudiantado. Discusión. La discusión sobre la evaluación educativa se focaliza en la crítica a los enfoques positivistastecnológicos que limitan la complejidad valorativa de los procesos de aprendizaje. Asimismo, se han relevado enfoques de evaluación para los aprendizajes como una forma de cuestionar y reinterpretar la teoría y práctica evaluativa positivista. Sin embargo, el debate ha marginado el rol de la evaluación en su dimensión ética-política para la construcción de sujetos democráticos. El argumento central sostiene que una evaluación transformadora y con un enfoque sociocultural-democrático posibilita la formación de sujetos participativos, comprometidos y reflexivos. Conclusiones. Se concluye con la importancia de resignificar la evaluación como una práctica no solo preocupada por la valoración de los aprendizajes, sino que también orientada a la formación de aspectos valóricos, actitudinales, éticos y democráticos, con la finalidad de contribuir a la construcción de un estudiantado consciente de sí mismo, de las otras personas y de su rol en la sociedad.
\end{abstract}

Palabras claves: Evaluación educativa; evaluación transformadora; evaluación formativa; evaluación para los aprendizajes. 
http://doi.org/10.15359/ree.25-3.40

http://www.una.ac.cr/educare

educare@una.ac.cr

\begin{abstract}
:
Purpose. In this article, educational evaluation is analyzed as a theoretical-practical field of knowledge for the democratic and transformative formation of students. Discussion. The discussion on educational evaluation focuses on criticism of positivist-technological approaches limited to the value complexity of learning processes. Likewise, they have relieved evaluation approaches for learning as a way to question and reinterpret positivist evaluative theory and practice. However, the debate has marginalized the role of evaluation in its ethical-political dimension for the construction of democratic subjects. The central argument argues that a transformative evaluation with a sociocultural and democratic approach enables the formation of participatory, committed, and reflective subjects. Conclusions. It concludes with the importance of re-signifying the evaluation as a practice not only concerned with the valuation of learning but also oriented to the formation of aspects in terms of values and attitudinal, ethical, and democratic aspects, in order to contribute to the construction of students aware of themselves, of others, and their role in society.
\end{abstract}

Keywords: Educational assessment; transformative assessment; formative assessment; assessment for learning.

\begin{abstract}
Resumo
Finalidade. Neste artigo, a avaliação educacional é analisada como um campo de conhecimento teórico-prático para a formação democrática e transformadora dos estudantes. Discussão. A discussão sobre avaliação educacional concentra-se na crítica de abordagens positivistas-tecnológicas limitadas à complexidade do valor dos processos de aprendizagem. Da mesma forma, foi revelado abordagens de avaliação da aprendizagem como uma maneira de questionar e reinterpretar a teoria e a prática avaliativas positivistas. No entanto, o debate marginalizou o papel da avaliação em sua dimensão ético-política para a construção de sujeitos democráticos. O argumento central afirma que uma avaliação transformadora e com uma abordagem sociocultural-democrática permite a formação de sujeitos participativos, comprometidos e reflexivos. Conclusões. É afirmada a importância de ressignificar a avaliação como prática, não apenas preocupada com o valor da aprendizagem, mas também orientada para a formação de valores, atitudes éticas e democráticas, a fim de contribuir para a construção de estudantes consciente de si mesmo, das demais pessoas e de seu papel na sociedade.
\end{abstract}

Palavras-chave: Avaliação educacional; avaliação transformadora; avaliação formativa; avaliação para aprendizagem.

\title{
Introducción
}

Los procesos evaluativos en los sistemas de educación enfrentan, en la actualidad, nuevos desafíos relacionados con la formación integral y el desarrollo de los aprendizajes vinculados a la transformación experimentada por la sociedad en el siglo XXI. Esta situación implica la necesidad de readecuar las racionalidades evaluativas para valorar el aprendizaje del estudiantado desde una perspectiva integral y dinámica, a partir de enfoques formativos que posibiliten construir procesos, procedimientos y protocolos evaluativos orientados a la formación del sujeto (Ahumada, 2005). 
http://doi.org/10.15359/ree.25-3.40

Una posible respuesta a estos desafíos del sistema educativo proviene de la política evaluativa que enfatiza la implementación de procesos evaluativos de calidad para el fortalecimiento de la enseñanza y aprendizaje (Murillo y Román, 2010). Estas iniciativas no solo buscan mejorar los desempeños del estudiantado, también instan a que los diferentes países avancen en el desarrollo de sus respectivos sistemas educativos con un triple foco de acción: la formación de aprendizajes orientados a la calidad, equidad e integración; la gestión escolar y la práctica docente y, finalmente, la apropiación de habilidades transversales o para la vida (Murillo y Román, 2010).

De esta manera, la evaluación para el aprendizaje se ha preocupado por posicionary relevar el rol de la formación integral del estudiantado en las instituciones escolares. Esto conlleva a la necesidad de profundizar diálogos y reinterpretaciones sobre el rol de la evaluación en el desarrollo de sociedades democráticas, diversas y con afán equitativo que trascienda su foco tradicional tyleriano: una evaluación formativa transformadora para la construcción de sujetos que asuman una acción crítica-reflexiva y humanizadora en la sociedad actual (Borjas, 2014).

En este contexto, la formación integral en los ámbitos educativos debe enfrentar las exigencias de sociedades que demandan mayores niveles de igualdad e inclusión y grados de participación y reestructuración de la institucionalidad democrática. Frente a esta situación crítica, la evaluación de los aprendizajes asume un rol primordial para readecuar y redefinir los nuevos desafíos educativos, con la finalidad de fomentar la construcción de sujetos comprometidos con el progreso social. Esto en la práctica significa alterar las lógicas tradicionales de la evaluación para incorporar nuevas prácticas evaluativas en el aula en sus diferentes niveles educativos. El fundamento es avanzar en la construcción de sujetos conscientes de sí mismos, de sus aprendizajes y de sus acciones en el mundo social (Fernandes, 2009). Así, la escuela y la evaluación están desafiadas para contribuir y cooperar en la formación de sujetos responsables y comprometidos con los valores democráticos para la consolidación de una convivencia ciudadana diversa y plural (Bonhomme et al., 2015).

Para enfrentar estos desafíos es necesaria la readecuación de las prácticas evaluativas con la finalidad de fortalecer la formación ciudadana. Tal como es planteado por Delors (1996), uno de los cuatro pilares de la educación se orienta al aprender a vivir en conjunto. Por lo tanto, la escuela se convierte en un espacio privilegiado para el desarrollo de una acción reflexivapedagógica que contribuya a este camino (Bonhomme et al., 2015).

Por esto, la evaluación es fundamental para favorecer la participación del estudiantado con el propósito de promover su autonomía y posibilitar la construcción de valores sociales para su reflexionar y actuar en democracia. Perspectivas como la evaluación auténtica, formativa, integral, socialmente justa y equitativa, transformativa y evaluación como compromiso, son enfoques que convergen para la formación democrática y transformadora de los sujetos en el sistema educativo (Ahumada, 2005; Borjas, 2014; Fernandes, 2009; Gipps y Stobart, 2003; Murillo e Hidalgo, 2018; Popham, 2013; Stiggins, 2004). 
http://doi.org/10.15359/ree.25-3.40

http://www.una.ac.cr/educare

educare@una.ac.cr

La autoevaluación, la coevaluación y la evaluación de pares son estrategias relevantes para promover la construcción del sujeto con un enfoque democrático y transformador de la formación. La autoevaluación se concibe como una acción autocrítica del sujeto para valorar su propio desempeño. La coevaluación es una valoración interpersonal del desempeño que involucra a docente-estudiantes y estudiantes entre sí. La evaluación de pares es la acción crítica y reflexiva entre estudiantes, según sus contribuciones individuales y colectivas sobre los desempeños logrados (Allal, 2020; Panadero et al., 2017).

La complejidad de estos procesos no solamente responde a la necesidad de objetivar procedimientos instrumentales para garantizar los aprendizajes, más bien, propone un desafío sobre cómo en el aula tradicional se incorporan prácticas de evaluación descentralizada que fomenten otras lógicas de participación, colaboración y autorreflexión. La evaluación descentralizada permite la redefinición de los roles entre docentes y estudiantes para la coconstrucción de aprendizajes en forma democrática y participativa, ya que se convierte en un espacio de negociación de significados, intercambio de conocimiento y fomento al diálogo y discusión de los procesos formativos (Ríos Muñoz y Herrera Araya, 2020). Esto implica una exigencia ética y responsabilidad de los sujetos para el desarrollo de estas formas evaluativas (Murillo e Hidalgo, 2018).

Estas acciones evaluativas contribuyen a la formación ética del estudiantado, entendida como un acto de reflexión, autoconciencia y conciencia con otros seres en un contexto justo y de responsabilidad social, orientada a la participación comprometida y basada en sus derechos y deberes, como un acto de formación socio-histórica, política y trascendental. En efecto, una racionalidad evaluativa naturalista-formativa, con una perspectiva transformadora sobre la evaluación y, desde un enfoque sociocultural democrático, permiten la construcción de una cultura evaluativa descentralizada por cuanto posibilita la reestructuración de concepciones, prácticas y fundamentos técnico-positivistas que son hegemónicos en las instituciones escolares (Ahumada, 2005; López-Pastor y Pérez-Pueyo, 2017). De esta manera, una evaluación democrática es una acción humanista-transformadora que redefine la práctica pedagógica y el rol del estudiantado para poner su foco de atención a la formación democrática y participativa de los sujetos. Es transformadora pues se posiciona como un espacio ético-moral que busca contribuir al aprender a convivir con otros.

Para abordar estas temáticas, el artículo se organiza en cinco apartados. El primero se preocupa por problematizar la educación como un proceso humanista-transformador del sujeto con una perspectiva social. El segundo, describe el rol de los actores escolares en el contexto de una evaluación transformadora. El tercero, busca esbozar las características y tensiones sobre una evaluación transformadora y su relación con la formación del sujeto democrático y ético. El cuarto, posiciona a la evaluación para el aprendizaje como un espacio de co-construcción de la formación integral basada en una evaluación formativa-transformadora. Por último, las 
http://doi.org/10.15359/ree.25-3.40

conclusiones están centradas sobre la importancia de prácticas evaluativas que releven el rol de los valores, actitudes y la toma de conciencia en los procesos formativos de la persona en pos de una ciudadanía crítica y reflexiva.

\section{La educación como proceso humanista-transformador: Tensiones para la evaluación}

La educación es esencialmente política y ética porque contribuye a la formación integral de las personas y, a través de la actuación de esta, al mejoramiento de nuestra sociedad, a partir de su integración crítica y propositiva, plena de derechos y deberes ciudadanos. No existe herramienta más poderosa que la educación para cumplir con esta finalidad: la formación transformadora del estudiantado (Ríos Muñoz y Herrera Araya, 2020). Como es sostenido por McDonald (1976), una evaluación democrática exige confidencialidad, negociación y accesibilidad por cuanto permite desafiar los monopolios de varios tipos: de definición de problemas, de formulación de cuestiones, de control de datos, de utilización de la información (MacDonald, 1978) y, por lo tanto, redefinir el rol de los sujetos en estos espacios evaluativos.

La función social de la evaluación posiciona su quehacer en el ámbito de la construcción de aprendizajes complejos para la vida. Esto implica las formas y modos de interacción social y de cómo la integración de sujetos diversos involucra replantearse las acciones escolares en tanto acciones socioculturales de transformación pedagógica. En efecto, no basta solo con apuntar al desarrollo de capacidades cognitivas superiores, también es necesario cuestionar las concepciones, normativas y técnicas sobre una práctica evaluativa estándar que no asume procesos de diversidad social y, menos, de los problemas asociados a los conflictos de distribución cultural en la escuela (House y Howe, 2001; Moreno-Olivos, 2010).

La educación y en particular la evaluación, presentan un sentido político en su constitución como espacio de formación. Esto se explica tanto por su impacto sobre la delimitación y definición de calidad de las instituciones educativas, como también por su acción en la formación del sujeto (Jiménez Moreno, 2019). Su tensión radica en la búsqueda de su valía como campo de conocimiento sobre los procesos de construcción del sujeto. A saber, se identifican dos tensiones: por una parte, como actividad política por su rol en la sistematización educativa y en la rendición de cuentas. Por otro, como anclaje cultural por cuanto a su práctica formativa-transformadora del aprendizaje desde una perspectiva humanista, integral y reflexiva (Carbajosa, 2011).

Por lo tanto, la función social de la educación en acción integrada a la evaluación como campo de conocimiento orientado a la construcción transformadora del sujeto es una actividad ético-moral (Moreno-Olivos, 2010), mediada por un anclaje cultural, sustentada en la interpretación de la acción humana y, sobre todo, contextualizada en los referentes socioculturales en los cuales se inserta, ya sea de la cultura escolar como del sistema educativo 
http://doi.org/10.15359/ree.25-3.40

http://www.una.ac.cr/educare

educare@una.ac.cr

y social en general (Jiménez Moreno, 2019). Así, la acción evaluativa como actividad éticamoral se transforma en el espacio de mediación pedagógica integradora en tanto evaluación democrática, ya que posibilita la reformulación del rol docente como formador crítico y autocrítico, y al estudiantado como sujeto autorreflexivo y activo de su formación para la vida.

Esta buena educación, indistintamente de los orígenes socioeconómicos y culturales del estudiantado, conlleva a la formulación de una serie de interrogantes sobre su delimitación y acción en la formación del sujeto. ¿Nuestras escuelas están respondiendo a estos desafíos que demanda la sociedad? Y si es así, ¿cómo estos desafíos se incorporan y dan vida a los proyectos educativos de cada institución escolar?, y por ende, ¿cómo el cuerpo docente está educando para que el estudiantado aprenda a convivir reconociendo a la otra persona? Por último, ¿el estudiantado está aprendiendo anclajes culturales fundamentales para su desarrollo personal y su contribución a la sociedad?

La paradoja se inserta en cómo comprender el valor y el juicio de la evaluación en una delimitación del deber justo y la responsabilidad moral. Así, la problemática es evidente: el ejercicio co-constructivo de una evaluación orientada a la formación ética del sujeto y, por otro, la complejidad de su origen como objeto de investigación y campo del saber, entendida como la búsqueda de neutralidad anclada en el valor de la objetividad para dar cuenta del aprendizaje en evaluaciones sobre el logro cognitivo y comparativo con los referentes y dispositivos curriculares (Tristán López y Pedraza Corpus, 2017). En contraposición, el camino es avanzar en integrar el fenómeno escolar y evaluativo desde procesos intersubjetivos como parte fundamental de la actuación valorativa y ética de una práctica evaluativa que permita el intercambio de significados con base en el juicio evaluativo negociado y compartido con reconocimiento de otro ser (Popham, 2013).

En suma, es posible preguntarse si la evaluación está al servicio de la formación plena del estudiantado y al servicio de una sociedad democrática.

\section{Los actores escolares: Co-construcción en el proceso evaluativo}

Desde una perspectiva sociocultural sobre la evaluación, los procesos de co-construcción se potencian al incorporar la participación de los sujetos en la dinámica evaluativa. Docentes como estudiantes son fundamentales para articular espacios de negociación de significados e intersubjetividades para avanzar en aprendizajes desde el deber sery, sobre todo, desde el convivir con otros seres. Aquí existe una responsabilidad compartida: por parte de docentes, por cuanto al desarrollo vital de la enseñanza-aprendizaje en la reflexión e implementación curricular y su gestión pedagógica en el aula. El estudiantado, como agente activo de sus aprendizajes que tensionan la práctica docente para profundizar la crítica, autocrítica y la formación consciente de su quehacer como sujeto individual y colectivo. 
http://doi.org/10.15359/ree.25-3.40

En este espacio el estudiantado puede aprender no solo conocimientos y habilidades, sino también aquellos comportamientos, valores y actitudes que en su futura construcción social pueden contribuir a su integración crítica, comprometida y activa participación en la sociedad. Desde el imperativo ético y los principios de diversidad y tolerancia, el liderazgo pedagógico orientado a colaborar al proceso formativo de estudiantes, se transforma en un horizonte de oportunidades para garantizar una buena educación. Esto posibilita una formación holística y sitúa a la evaluación como una acción articuladora entre los conocimientos, actitudes y habilidades que componen una formación para la vida.

Este marco interpretativo es fundamental. Asumir que la evaluación es un proceso formativo implica desafiar las funciones unívocas sobre el rol de los agentes escolares en su quehacer. Por tanto, es en su construcción teórica y en su acción explícita donde se evidencian posibilidades de analizar y reflexionar en una práctica consciente evaluativa y para esta, centrada en la interacción participativa y desafiante entre estudiantes y docentes (Rodríguez Migueles y Hernández Yulcerán, 2014; Román, 2011).

A partir de los postulados de Dewey (1916/1998), emerge una oportunidad para aprender y practicar la convivencia democrática. Para ello, es necesario que el personal docente tome conciencia de la importancia del liderazgo pedagógico para co-construir espacios de aprendizajes basados en un clima de aula democrático-participativo. Este se caracteriza por construir relaciones cordiales con sus estudiantes, respetarles como sujetos del proceso educativo en concordancia con un diseño de la enseñanza que articule las necesidades de los aprendizajes en prácticas evaluativas alternativas (Gipps y Stobart, 2003). Estas permiten incorporar las características sociales y culturales del estudiantado que se transforma en la base sustantiva de la diversidad democrática en el aula (Fernandes, 2009).

Ello nos lleva a la necesidad de resignificar el sentido de la escuela como espacio de co-construcción de aprendizajes transversales orientados a la formación de sujetos críticos/ autocríticos como un campo de construcción de ciudadanía. En efecto, la redefinición de la cultura evaluativa escolar como las prácticas implícitas y explícitas que constituyen la base de la construcción social en la escuela, demanda de la creación de espacios evaluativos sociodemocráticos que reconozcan y se articulen a partir de la reflexión sobre la experiencia, la argumentación y el diálogo (Bonhomme et al., 2015). Es decir, la evaluación puede contribuir en la creación de espacios participativos en función de acciones descentralizadas que permitan enriquecer el rol estudiantil con un alto grado de discusión democrática (Moreno-Olivos, 2010).

En este sentido, se hace imperativo hoy que los cuerpos docentes eduquen más que transmitan información, como una práctica robusta, relevante y contextualizada. Esta educación se sustenta en el pensar y el sentir docente. En lo racional y emocional como una fuente reflexiva (Maturana, 2001) para resignificar las interacciones de los aprendizajes y, por tanto, del mismo sentido de la evaluación. 
http://doi.org/10.15359/ree.25-3.40

http://www.una.ac.cr/educare

educare@una.ac.cr

\section{Hacia una evaluación transformadora}

Para orientar la práctica pedagógica es esencial la presencia de los objetivos escolares. En estos se plasman los conocimientos, habilidades y actitudes que el estudiantado debe alcanzar como producto de su experiencia educativa. Sin embargo, por decisiones curriculares conscientes o inconscientes de diferentes actores educativos, estos objetivos se reducen a la enseñanza del conocimiento y de las habilidades básicas. Esta situación provoca que la evaluación se reduzca al acto de medición psicométrica sobre el aprendizaje, lo que lleva a enfatizar la aplicación de evaluaciones más tradicionales desde una perspectiva positivistatécnica de la práctica evaluativa. En este panorama, se asume la evaluación desde la concepción tyleriana. Lo esencial en esta racionalidad y práctica es medir los resultados del aprendizaje de acuerdo con lo planteado en los objetivos con énfasis en los conocimientos reproductivos y habilidades de pensamiento superficiales (Ríos Muñoz y Herrera Araya, 2020).

Estas acciones se sustentan desde un principio básico: mucha evaluación, pero sin cambios (Moreno-Olivos, 2010). La medición como acción evaluativa instrumental no genera transformación, más bien, alimenta indicadores de mejora como un acto compulsivo que apunta a la burocratización del aprendizaje, en un proceso de adquisición que no cuestiona el qué se aprende y por qué se aprende (Shepard, 2006; Stobart, 2010). Así, las pruebas y las preguntas de selección múltiple son los principales artefactos que se utilizan para evaluar los aprendizajes. Esta perspectiva empobrece la misión política y ética de la educación, porque se tiende a valorar más un tipo de aprendizaje por sobre otros, como si estos fueran excluyentes. Se hacen invisibles, sobre todo, aquellos que justamente contribuyen a la formación integral del sujeto: valores y actitudes para la educación ciudadana. Esta reducción de complejidad sobre el aprendizaje pone en riesgo el bien común y la justicia social (House, 1997; Shepard, 2006; Stobart, 2010).

De esta forma, es importante consolidar prácticas evaluativas que coloquen en su centro al sujeto en su dimensión política-ética. Una perspectiva que lo resalte como sujeto sociohistórico; que lo considere como el principal responsable de la propia construcción personal, de sí mismo, pero también en relación con otras personas, sustentado en el conocimiento de su mundo social, con principios de respeto y reflexión (Maturana, 2001).

Para esto se requiere de una evaluación para el aprendizaje, una evaluación esencialmente educativa, que contribuya a generar una curiosidad y una motivación permanente en el estudiantado, en espacios escolares que le otorguen la posibilidad de vivir y practicar, lo que será su futuro comportamiento en sociedad (Dewey, 1916/1998). Este tipo de evaluación se constituye como un espacio descentralizado para visibilizar la desigualdad que genera el poder evaluativo concentrado en docentes. Esta asimetría coloca al personal docente en la cima de la montaña y al estudiantado en el valle. Aquí se pone en juego todo el poder que descansa en quienes educan, en sus juicios evaluativos, en las decisiones que toman a partir de las evidencias

8

Los artículos de la Revista Electrónica Educare del Centro de Investigación y Docencia en Educación de la Universidad Nacional, Costa Rica, se comparten bajo términos de la Licencia Creative Commons: Reconocimiento, No Comercial, Sin Obra Derivada 3.0 Costa Rica. Las autorizaciones adicionales a las aquí delimitadas se pueden obtener en el correo: educare@una.cr 
http://doi.org/10.15359/ree.25-3.40

que recogen. Por lo tanto, ¿es posible compartir este poder que entrega la evaluación?, ¿está dispuesto el personal docente a distribuir este poder? O planteado en positivo: ¿está dispuesto a compartirlo con sus estudiantes? ¿es posible que docentes y estudiantes caminen por valles para llegar en conjunto a la cima?

Debemos buscar las estrategias para descentralizar el acto evaluativo docente y, por ello, se hace necesario democratizar este proceso de valoración de los procesos de aprendizaje (Stake, 2006). Es un imperativo político y ético avanzar hacia una mayor simetría, sin perder los roles y funciones que conlleva la tarea pedagógica. En consecuencia, avanzar hacia una más amplia participación del estudiantado en los procesos valorativos (Stake, 2006) implica, tal como se ha planteado, la implementación de agentes de autoevaluación, coevaluación y evaluación entre pares.

Con una evaluación transformadora se potencia la co-construcción de sujetos activos que permite el desarrollo de una atmósfera evaluativa-formativa para la consolidación de una distribución del poder evaluativo y la participación crítica de docentes y estudiantes en el aula (Black y William, 1998; Fernandes, 2009; Stiggins, 2004). Una posibilidad para concretizar este enfoque es articular la evaluación transformadora-alternativa como un nodo crítico de la enseñanza-aprendizaje. Su reestructuración procura sustentarse en el diseño y selección de tareas auténticas que combinen lo disciplinar y la naturaleza transversal del aprendizaje por ejemplo, la dimensión socioafectiva, valórica y ética-, su transparencia ética-valorativa en función de los sujetos y, consolidar la retroalimentación como espacio dialógico dinámico que privilegia la co-participación y co-responsabilidad (Black y William, 2018; Fernandes, 2009; Gipps y Stobart, 2003; Popham, 2013).

Estos principios de reciprocidad conllevan al fortalecimiento de la autorreflexión y responsabilidad del estudiantado, lo cual lo conduce a una mayor autonomía y libertad. Así, es la acción comunicativa democrática un medio que permite, no solo consensuar estas definiciones, sino que también, promueve el entendimiento mutuo entre los sujetos. De esto trata la buena evaluación: de permitir el autogobierno y la construcción del sujeto en su dimensión particular como social; de avanzar hacia la autonomía y la libertad. Solo desde esta base puede construirse, en conjunto, una comunidad que busca dar cuenta del bien común. Para ello, se asume un principio básico articulador en la escuela: la existencia de un diálogo igualitario que favorezca esta construcción comunitaria (House, 1997; Santos Guerra, 2003).

\section{Evaluación para el aprendizaje}

Una herramienta que favorece el logro de estas finalidades se relaciona con la práctica evaluativa que efectúa el personal docente. Desde esta visión, ha cobrado relevancia la concepción de la evaluación para el aprendizaje. Esta hace énfasis en los procesos como una forma de consolidar el progreso de los aprendizajes. Este enfoque es naturalista y utiliza instrumentos 
http://doi.org/10.15359/ree.25-3.40

http://www.una.ac.cr/educare

educare@una.ac.cr

evaluativos basados en la observación. Su foco es relevar la participación estudiantil en su proceso evaluativo, mediante el desarrollo de juicios sobre su desempeño, sus avances y las condicionantes que favorecen o que impiden sus aprendizajes (Ahumada, 2005). Asimismo, se preocupa por integrar el rol de las actitudes que el estudiantado presenta durante el proceso de enseñanza-aprendizaje, transformandolo en sujeto y objeto evaluativo (Escudero Escorza, 2003, 2016; Ríos Muñoz y Herrera Araya, 2020).

La evaluación para el aprendizaje permite consolidar instancias de autovaloración que se sostienen con la toma de decisiones personales para la mejora del aprendizaje. Esta actividad autoevaluativa lo visibiliza como un sujeto constructor de sí mismo. Esta participación del estudiantado en el fenómeno evaluativo los visibiliza como sujeto, lo empodera como actor escolar, le genera la necesaria introspección y reflexión, para aprender a valorarse respecto a sus aprendizajes, le posibilita aprender a reconocer sus fortalezas y debilidades, a abstraerse como sujeto siendo objeto de su propia valoración (Santos Guerra, 2003). Estas son condiciones necesarias para su autoconstrucción. Este poder que adquiere y práctica es, sin ninguna duda, una valiosa experiencia educativa que será en parte la base de su futura actuación ciudadana.

Con esta perspectiva evaluativa, las acciones evaluativas se constituyen en espacios de empoderamiento estudiantil que invitan a la problematización del conocimiento, el rol de la criticidad y justicia evaluativa y las tensiones que implica la cooperación entre sujetos para una evaluación transformadora. Por lo tanto, aquí subyacen principios, desafíos y oportunidades para una evaluación de los aprendizajes con perspectiva transformadora que articula los procesos de subjetivación, simbolización y resignificación sobre el ser ciudadano (Popham, 2013). Este enfoque valorativo se favorece con prácticas de evaluación descentralizada vinculadas con prácticas de coevaluación y evaluación de pares (López-Pastor y Pérez-Pueyo, 2017; Rodríguez Migueles y Hernández Yulcerán, 2014) y su rol en los juicios de valor que producimos respecto a los otros (Popham, 2013).

La educación tiene un desafío irrenunciable en la formación del estudiantado respecto a ejercer justicia con los demás individuos. Este aprendizaje escolar después se pondrá en práctica en el entramado social en que participará el estudiantado como ciudadano. En este marco, surgen las siguientes interrogantes: ¿cuán justos somos, como personal docente, con la otredad? ¿cómo podemos contribuir a una mejor democracia al reconocer las diferencias con los otros actores educativos?

En la coevaluación, el estudiantado aprende a construir un juicio compartido sobre los desempeños logrados. Durante el proceso educativo ellos y ellas van recogiendo evidencias sobre su propia actuación. Finalizada la actividad, el estudiantado está en condiciones de establecer diálogos que favorezcan el intercambio de significados con sus pares y de construir juicios valorativos sobre la actuación colectiva. En esta actividad evaluativa es clave que los juicios evaluativos se fundamenten en las evidencias observadas, y no a partir de juicios preexistentes o prejuicios de miembros participantes (Murillo e Hidalgo, 2018; Stobart, 2010). 
Desde lo valórico, la construcción del juicio asume una perspectiva justa de acuerdo con la evidencia del aprendizaje. De no tener sólidas evidencias, se debe suspender el juicio. Así, podemos preguntarnos: ¿es posible promover este tipo de práctica evaluativa en el aula?, ¿de qué manera contribuye a la descentralización del poder evaluativo?, ¿esta práctica evaluativa favorece la formación en valores como la responsabilidad, honestidad y justicia por parte del estudiantado?

El proceso de evaluación de pares constituye otra oportunidad educativa que tiene el estudiantado para practicar valores y actitudes de responsabilidad, honestidad y justicia en relación con sus compañeras y compañeros. A diferencia de la coevaluación, basada en la simetría entre estudiantes en interacción con la persona docente, la evaluación de pares se fundamenta en la asimetría, ya que el estudiantado evaluador se sitúa como un sujeto que elabora los juicios de valor sobre el par, cuyo desempeño o actuación es asumido como objeto evaluado (Santos Guerra, 2003; Spiller, 2012).

Esta heteronomía conlleva la responsabilidad de contar con la mayor cantidad de evidencias para levantar valoraciones sobre el quehacer del estudiante evaluado. Al igual que la autoevaluación y la coevaluación, en este proceso se despliegan todas posibilidades de practicar los valores de responsabilidad, honestidad y justicia, aprendizajes que en el futuro podrían extrapolarse al comportamiento ciudadano del estudiantado involucrado en estos procesos evaluativos.

\section{A modo de conclusión: Tensiones y desafíos para una evaluación formativa democrática y transformadora}

Para finalizar, y asumiendo los planteamientos centrales de este trabajo, se postula que la educación por definición es política y ética. Su misión es posibilitar el aprendizaje del estudiantado. No solo puede estar focalizada en el conocimiento y las habilidades cognitivas para desarrollar el pensamiento comprensivo del estudiantado. Sino que, se hace necesario promover e implementar actividades curriculares relacionadas con los valores y las actitudes de este.

Una evaluación democrática y transformadora permite la redefinición y el desplazamiento de los límites impuestos por enfoques evaluativos tecnológicos-positivistas. Reformula los roles de docentes y estudiantes, ya que se convierte en un espacio para la discusión y negociación de significados sobre los conocimientos, habilidades y actitudes que nutren el proceso formativo. En efecto, las acciones evaluativas se constituyen en espacios de evaluación descentralizada orientadas al empoderamiento docente y estudiantil en forma equitativa, por cuanto permite la problematización, incide en la criticidad y justicia evaluativa y tensiona las lógicas individuales en la construcción de aprendizajes y las sitúa en lógicas de cooperación evaluativa.

La acción evaluativa es una actividad ético-moral que se transforma en una mediación pedagógica que busca la integración de la evaluación democrática y transformadora para el desarrollo de prácticas evaluativas críticas, autocríticas, activas y autorreflexivas como un 
http://doi.org/10.15359/ree.25-3.40

http://www.una.ac.cr/educare

educare@una.ac.cr

horizonte real para el cambio de la cultura evaluativa escolar. Por lo tanto, la participación del estudiantado en los procesos evaluativos busca el desarrollo de la autonomía y libertad del sujeto. Así, la autoevaluación, la coevaluación y la evaluación de pares pueden constituirse en estrategias fundamentales para promover valores y actitudes que permita al estudiantado un actuar ético, con vista a su futura participación, crítica y propositiva en la sociedad a la cual pertenece.

Esta perspectiva nos invita a la toma de consciencia sobre el potencial peligro de la evaluación sobre el sujeto y sus efectos contradictorios en la formación. Para enfrentar estos desafíos evaluativos, es urgente reestructurar las lógicas del poder y autoridad en el aula y potenciar procesos de aprendizaje formativos desde principios éticos y morales alternativos a los actuales. Provistos de lógicas inclusivas y, sobre todo, desde el respeto a la diversidad, debe avanzarse en una mirada reflexiva cuya finalidad -como acto evaluativo- es primordialmente formativa y orientada al desarrollo de componentes ético-morales para la construcción de sujetos críticos y comprometidos con su comunidad.

Esto nos lleva a plantear las siguientes interrogantes sobre cómo avanzar en este camino: ¿Los cuerpos docentes están motivados para promover y acompañar este tipo de prácticas evaluativas? ¿Es posible que estás prácticas evaluativas contribuyan a generar una cultura evaluativa basada en la participación, colaboración, responsabilidad, honestidad y justicia? ¿Estas prácticas evaluativas permiten el desarrollo de ética en el estudiantado?

Considerando estas inquietudes y tensiones, el desafío de una evaluación orientada a la construcción democrática y transformadora del sujeto requiere de la construcción de culturas evaluativas que conciban y determinen la acción evaluativa como un acto ético-moral y formador del sujeto. Esta se relaciona con la acción humana y se enmarca en los contextos socioculturales del estudiantado. Avanzar en este proceso de consciencia y autoconciencia sobre los procesos de aprendizaje desde lo actitudinal y valórico es el horizonte de una evaluación que colabora con el fortalecimiento de la educación y del desarrollo social.

\section{Declaración de Material complementario}

Este artículo tiene disponible, como material complementario:

-La versión preprint del artículo en https://doi.org/10.5281/zenodo.3923115

\section{Referencias}

Ahumada, P. (2005). La evaluación auténtica: Un sistema para la obtención de evidencias y vivencias de los aprendizajes. Perspectiva Educacional, (45), 11-24. http://www.euv.cl/ archivos_pdf/rev_perspectiva_educ/persp_45_1sem.pdf 
http://doi.org/10.15359/ree.25-3.40 http://www.una.ac.cr/educare educare@una.ac.cr

Allal, L. (2020). Assessment and the co-regulation of learning in the classroom. Assessment in Education: Principles, Policy \& Practice, 27(4), 332-349. https://doi.org/10.1080/096959 $\underline{4 X .2019 .1609411}$

Black, P. y Wiliam, D. (1998). Assessment and classroom learning. Assessment in Education: Principles, Policy \& Practice, 5(1), 7-74. https://doi.org/10.1080/0969595980050102

Black, P. y Wiliam, D. (2018). Classroom assessment and pedagogy. Assessment in Education: Principles, Policy\&Practice,25(6),551-575.https://doi.org/10.1080/0969594X.2018.1441807

Bonhomme, M., Cox, C., Tham, M. y Lira, R. (2015). La educación ciudadana escolar de Chile 'en acto': Prácticas docentes y expectativas de participación política de estudiantes. En C. Cox y J. C. Castillo (Eds.), Aprendizaje de la ciudadanía. Contextos, experiencias y resultados (pp. 373-428). Universidad Católica de Chile.

Borjas, M. P. (2014). Educación y evaluación: Profecía o predicción. Revista Electrónica Educare, 18(3), 273-284. http://dx.doi.org/10.15359/ree.18-3.17

Carbajosa, D. (2011). Debate desde paradigmas en la evaluación educativa. Perfiles Educativos, 33(132), 183-192. https://doi.org/10.22201/iisue.24486167e.2011.132.24903

Delors, J. (Presidente). (1996). La educación encierra un tesoro. Ediciones UNESCO. http:// repositorio.minedu.gob.pe/handle/20.500.12799/1847

Dewey, J. (1916). Democracia y educación. Una introducción a la filosofía de la educación. Morata. Trabajo original publicado en 1998.

Escudero Escorza, T. (2003). Desde los tests hasta la investigación evaluativa actual. Un siglo, el $X X$, de intenso desarrollo de la evaluación en educación. RELIEVE, 9(1), 11-43. https://ojs. uv.es/index.php/RELIEVE/article/view/4348/4025

Escudero Escorza, T. (2016). La investigación evaluativa en el siglo XXI: Un instrumento para el desarrollo educativo y social cada vez más relevante. RELIEVE, 22(1), 1-20. https://doi. org/10.7203/relieve.22.1.8164

Fernandes, D. (2009). Avaliar para aprender. Fundamentos, prácticas e políticas. Editora UNESP.

Gipps, C. y Stobart, G. (2003). Alternative assessment. En T. Kellaghan y D. L. Stufflebeam (Eds.), International handbook of educational evaluation (549-576). Kluwer. https://doi. org/10.1007/978-94-010-0309-4 33

House, E. R. (1997). Evaluación, ética y poder. Morata.

House, E. R. y Howe, K. R. (2001). Valores en evaluación e investigación social. Morata. 
http://doi.org/10.15359/ree.25-3.40

http://www.una.ac.cr/educare

educare@una.ac.cr

Jiménez Moreno, J. A. (2019). Aproximaciones epistemológicas de la evaluación educativa: Entre el deber ser y lo relativo. Foro de Educación, 17(27), 185-202. http://dx.doi.org/10.14516/fde.636

López-Pastor, V. M. y Pérez-Pueyo, A. (Coords.) (2017). Buenas prácticas docentes. Evaluación formativa y compartida en educación: Experiencias de éxito en todas las etapas educativas. Universidad de León.

Maturana, H. (2001). Emociones y lenguaje en educación y política. Dolmen.

Moreno-Olivos, T. (2010). Lo bueno, lo malo y lo feo: Las muchas caras de la evaluación. Revista de Educación Superior, I(2), 84-97. https://doi.org/10.22201/iisue.20072872e.2010.2.6

MacDonald, B. (1976). 7 Evaluation and the control of education. En D. A. Tawney (Ed.), Curriculum evaluation today: Trends and implications (pp. 125-136). London: Macmillan.

MacDonald, B. (1978). Evaluation and democracy. Public address at the University of Alberta Faculty of Education.

Murillo, F. J., e Hidalgo, N. (2018). Concepciones de los docentes sobre la evaluación socialmente justa. Aula Abierta, 47(4), 441-448. https://doi.org/10.17811/rifie.47.4.2018.441-448

Murillo, F. J. y Román, M. (2010). Retos en la evaluación de la calidad de la educación en América Latina. Revista lberoamericana de Educación, 53, 97-120. https://doi.org/10.35362/rie530559

Panadero, E., Jonsson, A. y Botella, J. (2017). Effects of self-assessment on self-regulated learning and self-efficacy: Four meta-analyses. Educational Research Review, 22, 74-98. https://doi. org/10.1016/j.edurev.2017.08.004

Popham, W. J. (2013). Evaluación trans-formativa. El poder transformador de la evaluación formativa. Narcea.

Ríos Muñoz, D. y Herrera Araya, D. (2020). Decentralizing the assessment practice for student selflearning. Educação e Pesquisa, 46, 1-15.https://doi.org/10.1590/S1678-4634202046219544

Rodríguez Migueles, A. y Hernández Yulcerán, A. (2014). Desmitificando algunos sesgos de la autoevaluación y coevaluación en los aprendizajes del alumnado. REXE: Revista Estudios y Experiencias en Educación, 13(25), 13-31. http://www.rexe.cl/ojournal/index.php/rexe/ article/view/45

Román, M. (2011). Autoevaluación: Estrategia y componente esencial para el cambio de la mejora escolar. Revista lberoamericana de Educación, 55(1), 107-136. https://doi.org/10.35362/rie550527

Santos Guerra, M. Á. (2003). Dime cómo evalúas y te diré qué tipo de profesional y de persona eres. Revista Enfoques Educacionales, 5(1), 69-80. https://doi.org/10.5354/07173229.2003.47513 
http://doi.org/10.15359/ree.25-3.40

http://www.una.ac.cr/educare educare@una.ac.cr

Shepard, L. A. (2006). La evaluación en el aula. Instituto Nacional para la Evaluación de la Educación. https://www.academia.edu/6474331/La evaluaci\%C3\%B3n en el aula LA EVALUACI\%C3\%93N EN EL AULA

Spiller, D. (2012). Assessment matters: Self-assessment and peer assessment. University of Waikato.

Stake, R. E. (2006). Evaluación comprensiva y evaluación basada en estándares. Graó.

Stiggins, R. (2004). New assessment beliefs for a new scholl mission. Phi Delta Kappan, 86(1), 2227. https://doi.org/10.1177/003172170408600106

Stobart, G. (2010). Tiempos de pruebas: Los usos y abusos de la evaluación. Morata.

Tristán López, A., Pedraza Corpus, N. Y. (2017). La objetividad en las pruebas estandarizadas. Revista Iberoamericana de Evaluación Educativa, 10(1), 11-31. https://doi.org/10.15366/ riee2017.10.1.001 Luptat ende omnihit ianimin nobitaqui aborro que doluptas auda sint, sendignam qui doluptae etur? 